\title{
Preface to the Special Issue on Computing and Combinatorics
}

\author{
Yixin $\mathrm{Cao}^{1} \cdot$ Jianer Chen ${ }^{2}$
}

Published online: 23 September 2019

๑) Springer Science+Business Media, LLC, part of Springer Nature 2019

This special issue consists of five papers, selected from 48 presentations at the $23 \mathrm{rd}$ International Computing and Combinatorics Conference (COCOON 2017), taken place in Hong Kong, China, during August 3-5, 2017. COCOON 2017 provided a forum for researchers working in the areas of algorithms, theory of computation, computational complexity, and combinatorics related to computing. The technical program of the conference included 48 contributed papers selected by the Program Committee from 111 full submissions received in response to the call for papers. The Program Committee selected seven outstanding papers for a special issue in Algorithmica. After the regular peer-review process of the journal, five of them are accepted:

- Approximation Algorithms for the Maximum Weight Internal Spanning Tree Problem, by Zhi-Zhong Chen, Guohui Lin, Lusheng Wang, Yong Chen, and Dan Wang.

- Information Complexity of the AND Function in the Two-Party and Multi-party Settings, by Yuval Filmus, Hamed Hatami, Yaqiao Li, and Suzin You.

- Optimal Online Two-Way Trading with Bounded Number of Transactions, by Stanley P. Y. Fung.

- Hardness and Structural Results for Half-Squares of Restricted Tree Convex Bipartite Graphs, by Hoang-Oanh Le and Van Bang Le.

- Maximum Matching on Trees in the Online Preemptive and the Incremental Graph Models, by Sumedh Tirodkar and Sundar Vishwanathan.

One of the invited paper remains in review at this writing; if accepted, it will appear in a later issue of the journal.

We would like to express our gratitude to all reviewers for their hard work and the authors for their contribution. We would also like to thank Professor Ming-Yang Kao,

\footnotetext{
$\bowtie$ Yixin $\mathrm{Cao}$

yixin.cao@polyu.edu.hk

Jianer Chen

chen@cse.tamu.edu

1 Department of Computing, Hong Kong Polytechnic University, Hong Kong, China

2 Department of Computer Science and Engineering, Texas A\&M University, College Station, TX 77843, USA
} 
Editor-in-Chief, and Austin Hutchinson, Publishing Editor, of the Journal Algorithmica, for the great support and help in preparing this special issue.

Publisher's Note Springer Nature remains neutral with regard to jurisdictional claims in published maps and institutional affiliations. 